\title{
SEXUAL DYSFUNCTION AMONG WOMEN WITH BREAST CANCER DIAGNOSTIC: DESCRIPTIVE STUDY
}

Mayara M. P. Carvalho', Ana Luiza L. Sousa¹, Marcia F. Veloso', Ruffo Freitas-Junior¹

${ }^{1}$ CORA - Centro Avançado de Diagnóstico da Mama, Hospital das Clínicas, Universidade Federal de Goiás - Goiânia (GO), Brazil.

Backgroud: Studies about women's sexuality run into the lack of common sense concerning the adequate model of evaluation. In the presence of morbidity, such as breast cancer, in which physical and biological changes may happen due to treatment, this evaluation may reflect the way women face the disease. Objective: Describing women's sexual dysfunction in the moment they receive the diagnostic for breast cancer. Method: Descriptive study held in a reference support center. The Female Sexual Function Index Questionnaire (FSFI), in which 19 questions evaluated the sexual function during the last month, including the following domains: sexual desire, sexual arousal, vaginal lubrication, orgasm, sexual satisfaction, and pain. Reliability analysis was held by using Cronback`s alpha, categorized age group above and below 50 years old; the cut-off was between 25.66 and 22.80 according to the total FSFI scale for the dysfunction diagnostic. All of the participants were volunteers and freely took part in this study. Results: 42 women participated in the study, being the mean age 48.2 ( \pm 11.5 ), 26 minimum and 72 maximum. The group ages were similarly distributed. The instrument reliability was 0.901 . We identified 35 (83.3\%) and 25 (59.5\%) women with sexual dysfunction (cut-off 25.66 and 22.8 respectively). There was not any differences in the distribution of the results according to group age. The lowest scores were in the following domains: sexual desire $(2.5 ; \pm 1.0)$, sexual arousal $(3.0 ; \pm 1.0)$. On the other hand, the highest ones were "pain" $(4.6 ; \pm 1.7)$ and vaginal lubrication $(4.1 ; \pm 1.2)$. There was negative and moderated parity between the domains "satisfaction" and the woman's age $(-0.341 ; \mathrm{p}=0.031)$. Conclusion: Women's sexual dysfunction showed high prevalence on women with breast cancer diagnostic, even though they had claimed having sexual satisfaction. The instrument cut-off range must be taken into account. 